\title{
ARTICLE
}

Received 19 Dec 2012 | Accepted 21 Mar 2013 | Published 23 Apr 2013 | Updated 7 Nov 2013

DOI: $10.1038 /$ ncomms2783

\section{Another rapid event in the carbon-14 content of tree rings}

Fusa Miyake ${ }^{1}$ Kimiaki Masuda ${ }^{1} \&$ Toshio Nakamura ${ }^{2}$

Previously, we have observed that the atmospheric ${ }^{14} \mathrm{C}$ content measured in tree rings showed a strong increase from AD 774 to 775 . Although the cause of this event can be explained by a large solar proton event or a short gamma-ray burst, a more detailed discussion of the cause is difficult because the rate of occurrence of such rapid ${ }^{14} \mathrm{C}$ events remains unknown. Here we report new ${ }^{14} \mathrm{C}$ measurements from $A D 822$ to 1021 , and the discovery of a second rapid increase of ${ }^{14} \mathrm{C}$ content from AD 993 to 994 . The ${ }^{10} \mathrm{Be}$ flux in the Antarctic ice core shows peaks corresponding to these two ${ }^{14} \mathrm{C}$ events. The proportions of flux increase $\left({ }^{14} \mathrm{C} /{ }^{10} \mathrm{Be}\right)$ of the two events are consistent with each other. Therefore, it is highly possible that these events have the same origin. Considering the occurrence rate of ${ }^{14} \mathrm{C}$ increase events, solar activity is a plausible cause of the ${ }^{14} \mathrm{C}$ increase events.

\footnotetext{
${ }^{1}$ Solar-Terrestrial Environment Laboratory, Nagoya University, Chikusa-ku, Nagoya 464-8601, Japan. ${ }^{2}$ Center for Chronological Research, Nagoya University, Chikusa-ku, Nagoya 464-8601, Japan. Correspondence and requests for materials should be addressed to F.M. (email: fmiyake@stelab.nagoya-u.ac.jp).
} 
$\mathrm{R}$ adiocarbon ${ }^{14} \mathrm{C}$ is produced in the Earth's atmosphere by nuclear interactions with galactic cosmic rays, most of which are charged particles. The flux of cosmic rays is modulated by the solar magnetic activity and the geomagnetic field. Radiocarbon oxidizes in the atmosphere to form ${ }^{14} \mathrm{CO}_{2}$ and is taken up by trees as a part of the global carbon cycle. As ${ }^{14} \mathrm{C}$ is a radioisotope with a half-life of 5,730 years, the ${ }^{14} \mathrm{C}$ content in tree rings provides a record of cosmic ray intensity and solar activity over a few tens of millennia.

While the solar magnetic activity and the geomagnetic field modulate the cosmic ray background, high-energy phenomena, such as gamma-ray events and large solar proton events (SPEs), can produce a large number of cosmic rays all at once. Then, if such events have occurred in the past, the ${ }^{14} \mathrm{C}$ content in tree rings is possible to record the rapid increase due to the events.

Recently, we found a rapid increase in ${ }^{14} \mathrm{C}$ content within 1 year from AD 774 to 775 (ref. 1). The possible cause of the event is a supernova or a large SPE. However, neither a local supernova nor a large SPE is likely responsible, because of a lack of record of corresponding supernova or supernova remnant and too high energy for SPE (ref. 1). There have been some attempts to specify the cause of the AD 775 event. Melott and Thomas ${ }^{2}$ have reexamined the flare energy of the AD 775 event by assuming a directional flare with opening angle of $24^{\circ}$, and concluded that implied energy of SPE is reduced to $\sim 10^{33} \mathrm{erg}$. This is about $1 /$ 100 energy of our calculation ${ }^{1}$, which presumes that flare particles propagated isotropically. Then SPE appears to be the possible cause of the AD 775 event $^{2}$. Some studies also show the SPE origin $^{3,4}$. On the other hand, although we considered only the normal supernova origin ${ }^{1}$, Hambaryan and Neuhauser ${ }^{5}$ claim that a short gamma-ray burst $(\mathrm{GRB})(<2 \mathrm{~s})$ can explain the $\mathrm{AD}$ 775 event. Although the cause of this event can be explained by a large $\mathrm{SPE}^{2-4}$ or a short $\mathrm{GRB}^{5}$, a more detailed discussion of the cause is difficult because the rate of occurrence of such rapid ${ }^{14} \mathrm{C}$ events remains unknown. We need to know whether more events similar to the AD 775 event exist in the past ${ }^{14} \mathrm{C}$ record. Although the ${ }^{14} \mathrm{C}$ increment around $\mathrm{AD} 775$ is the largest class in the last three millennia from IntCal09 data (which is a data set back to about 25,000 years $\mathrm{BP})^{6}$, there are a large number of period when the ${ }^{14} \mathrm{C}$ content has not been measured with 1 -year resolution. Therefore, it is possible that smaller increases than the AD 775 event are hidden in these unmeasured periods. These small increases will not be detected until after 1-year resolution measurements.

Here, we report the measurement of ${ }^{14} \mathrm{C}$ content during an extended period from AD 822 to 1021 to search other ${ }^{14} \mathrm{C}$ increase events, and found another rapid increase from AD 993 to 994. Considering the occurrence rate of the ${ }^{14} \mathrm{C}$ increase events, the large SPE is a plausible cause of the ${ }^{14} \mathrm{C}$ events.

\section{Results}

Measurement data. We collected a new series of biennial measurements of ${ }^{14} \mathrm{C}$ content in the Japanese cedar tree (Cryptomeria japonica), which is the same tree as that used for the $\mathrm{AD} 775$ event $^{1}$, from $\mathrm{AD} 822$ to 1021 . In addition to these measurements, we also measured annual resolution data from AD 991 to 1001 in the same tree. The information about the sample is shown in Supplementary Table S1 and Supplementary Fig. S1. As the ${ }^{14} \mathrm{C}$ data for AD 600 to 820 have been already measured ${ }^{1}$ (Miyake, F., Masuda, K. and Nakamura, T. manuscript in preparation), we obtained a continuous series of AD 600 to 1021 . We have updated our previous series ${ }^{1}$ by measuring new data (for even years: AD $752-768,782-788$ and 794-820, and for odd years: AD 767 and 769). As data for AD 896 and 898 do not exist, we obtained values of ${ }^{14} \mathrm{C}$ content (which is described as $\Delta^{14} \mathrm{C}$ (ref. 7) for those years by a linear interpolation method. As the data for overlapping years match within measurement errors, these series of measurements are reproducible and we applied weighted averaging for the same year. Figure 1 shows the consolidated $\Delta^{14} \mathrm{C}$ data of some series for the period of AD 600 to 1021 .

The first half of Fig. 1 shows a grand solar minimum from AD 650 to 720 (Miyake, F., Masuda, K. and Nakamura, T. manuscript in preparation) and a rapid increase of ${ }^{14} \mathrm{C}$ content from $\mathrm{AD} 774$ to 775 (ref. 1). The second half of Fig. 1 shows a rapid increase of $9.1 \%$ in ${ }^{14} \mathrm{C}$ content from $\mathrm{AD} 993$ to 994 . This is a clear increase that is outside the measurement error $(5.1 \sigma)$. A comparison of the $\mathrm{AD} 775$ and the AD 994 events is shown in Supplementary Table S2 and Fig. 2. The shapes of the two series are very similar, that is, a rapid increase within 1 year followed by a decay owing to the carbon cycle. The scale of the AD 994 event is 0.6 times as large as the AD 775 event.

Comparison with IntCal data set. Although the quasi-decadal IntCal09 data set ${ }^{6}$ shows a small ${ }^{14} \mathrm{C}$ enhancement around $\mathrm{AD}$ 994 (3 permil increase from AD 980 to 995), this increase is hardly distinguishable from many other variations. To compare our results (obtained from Japanese trees) with IntCal98 (obtained from North American and European trees) ${ }^{8}$, we averaged the yearly data to obtain a series with a decadal time resolution. The result from $\mathrm{AD} 605$ to 1015 is shown in Fig. 3. The two series show similar variations to each other; however, our results are 2.1 permil smaller than IntCal98 on average (this is $7.0 \sigma$ significance level). This is considered as a regional effect. As Yaku-Island, where our sample trees lived, is located in southern Japan and is surrounded by ocean, its atmosphere is affected by marine low ${ }^{14} \mathrm{C}$ level gas ${ }^{9}$.

\section{Discussion}

The ${ }^{14} \mathrm{C}$ content measurements for the recorded ages of supernova explosions (SN1006, SN1054, SN1572, SN1604 and SN1885) (refs 10-12) and the emergence years of large solar flares (the Carrington flare (SPE1859) that occurred in $\mathrm{AD} 1859$, and SPE1460 that was detected by annual ${ }^{10} \mathrm{Be}$ data ${ }^{3,13,14}$ ) (refs 12,15,16) have been conducted (as shown in Supplementary

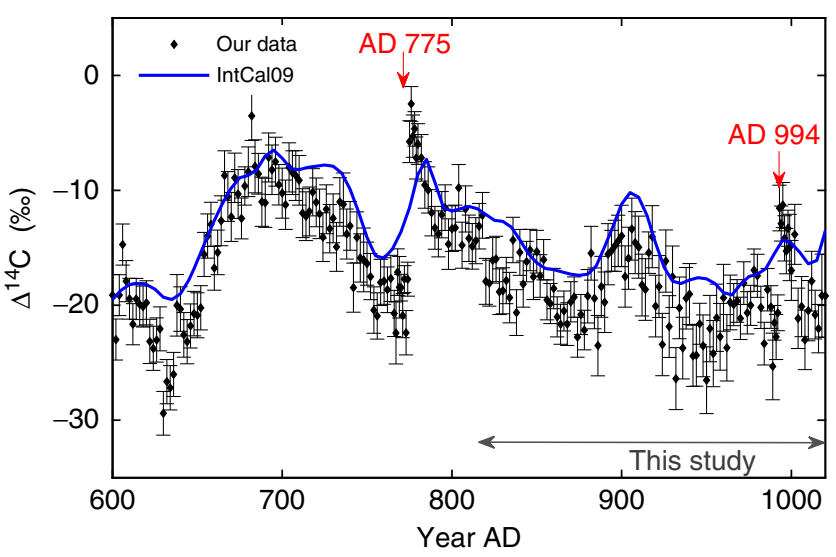

Figure $1 \mid{ }^{14} \mathrm{C}$ data from AD 600 to 1021. Diamonds show our results, which are obtained by a weighted mean of the 2-5 series. The blue curve represents the IntCal09 data set ${ }^{6}$. The errors are the resultant of error propagation. The error for a sample is a statistical error from a Poisson distribution, and the error for the standard sample is the greater one of either averaged statistical error from Poisson distribution of $\Delta^{14} \mathrm{C}$ for the six standard samples or the s.d. of values of ${ }^{14} \mathrm{C} /{ }^{12} \mathrm{C}$ for six standard samples. 


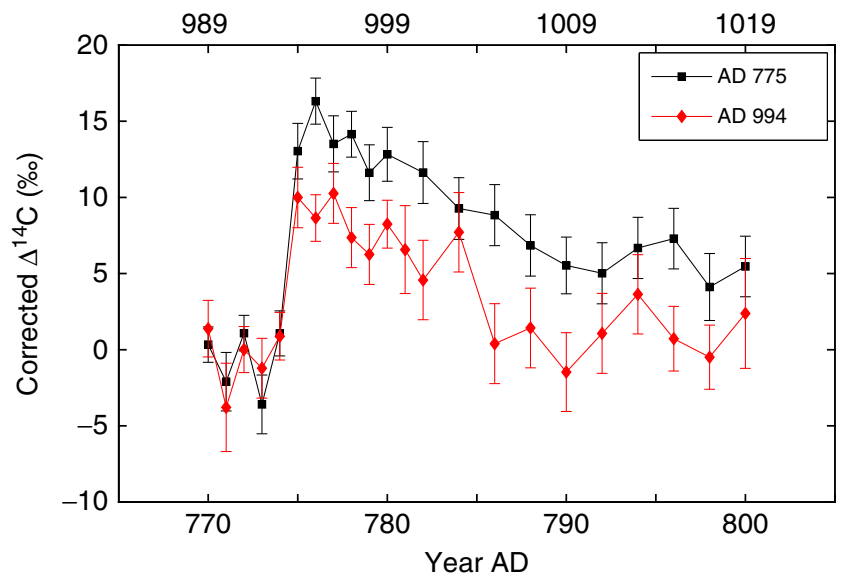

Figure 2 | Comparison of the AD $\mathbf{7 7 5}$ and the AD $\mathbf{9 9 4}$ peaks. Squares show the AD 775 series from AD 770-800, and diamonds show the AD 994 series from AD 989-1019. The zero level of the vertical axes is shifted to be the weighted mean value of $A D 770-774$ for the $A D 775$ series and AD 989-993 for the AD 994 series. The errors are the resultant of error propagation. The error for a sample is a statistical error from a Poisson distribution, and the error for the standard sample is the greater one of either averaged statistical error from Poisson distribution of $\Delta^{14} \mathrm{C}$ for the six standard samples or the s.d. of values of ${ }^{14} \mathrm{C} /{ }^{12} \mathrm{C}$ for six standard samples.

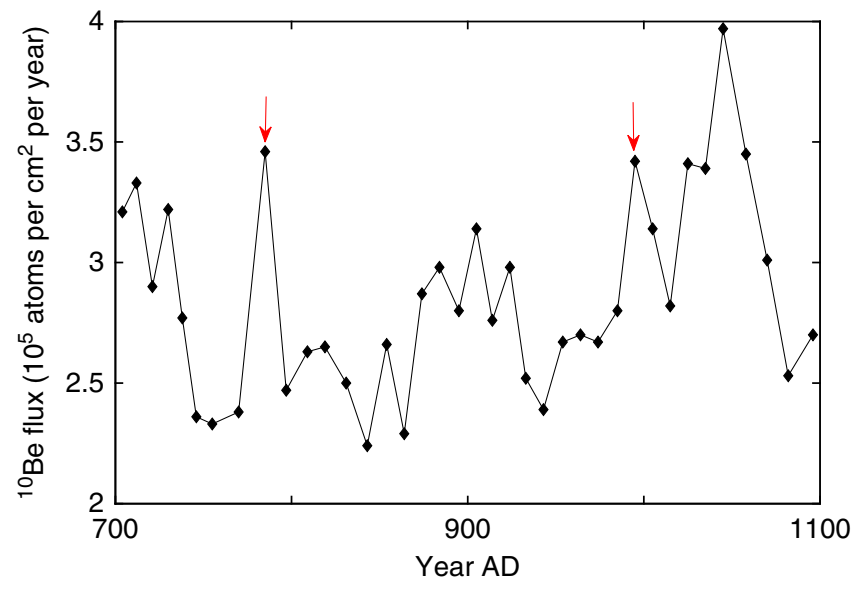

Figure 4 | ${ }^{10} \mathrm{Be}$ data from ice core of Dome Fuji in Antarctica. The vertical axis represents the ${ }^{10} \mathrm{Be}$ flux, which is calculated from the snow accumuration rate, estimated by the three-point (1.5 m: 30 years) averaged $\delta^{18} \mathrm{O}$ (ref. 5). The horizontal axis represents the calendar year. Each point is corrected by a ${ }^{10} \mathrm{Be}-{ }^{14} \mathrm{C}$ correlation age model. The two arrows show the ages of $A D 785$ and $A D$ 995. The ${ }^{10} \mathrm{Be}$ flux increments of the two data is $1.08 \times 10^{5}$ (atoms per $\mathrm{cm}^{2}$ per year) from AD 770 to 785 and is $0.72 \times 10^{5}$ (atoms per $\mathrm{cm}^{2}$ per year) from AD 985 to 995 .

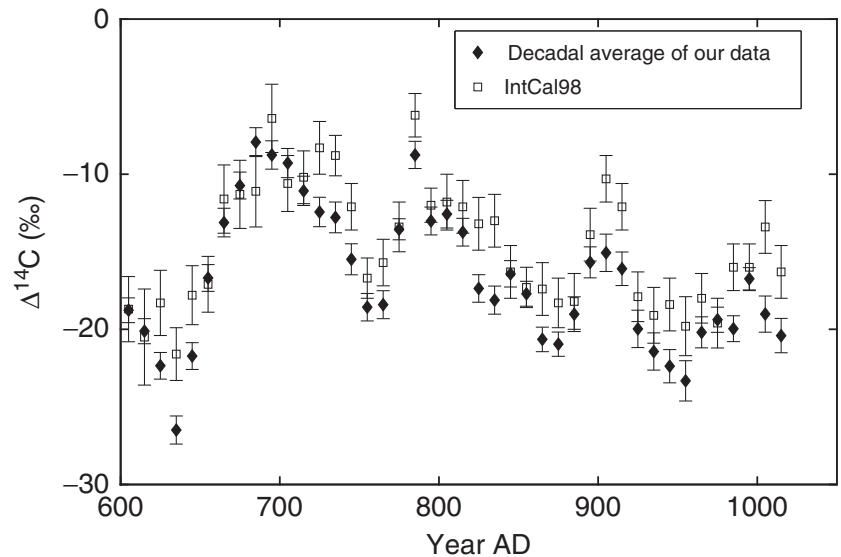

Figure 3 | Comparison of our result with IntCal98 data. The vertical axis represents the ${ }^{14} \mathrm{C}$ content (in $\Delta^{14} \mathrm{C}$ ), and the horizontal axis represents the calendar year. Open squares show the IntCal98 data and filled diamonds show the decadal average of our data (AD 600-1021).

Fig. S2). We also measured the ${ }^{14} \mathrm{C}$ content around $\mathrm{SN} 1054$ and SPE1859 using another Japanese cedar tree (Tree-C, information of this sample is shown in Supplementary Table S1). However, none of the ${ }^{14} \mathrm{C}$ contents show rapid increases within 1 year. There is no ${ }^{14} \mathrm{C}$ increase within 1 year during other periods, from $\mathrm{AD} 1374$ to 1954 (refs $12,15-17$ ). Only rapid ${ }^{14} \mathrm{C}$ increases within 1 year in $\mathrm{AD} 775$ and 994 have been found during about 1,600 years (when we have 1- or 2-year resolution ${ }^{14} \mathrm{C}$ data).

Another cosmogenic nuclide, ${ }^{10} \mathrm{Be}$, in the Antarctic Dome Fuji ice core also shows increases in the flux corresponding to around AD 775 and AD 994 (ref. 18). Figure 4 shows ${ }^{10} \mathrm{Be}$ flux data for $\mathrm{AD} 700-1100$. The ages of the ${ }^{10} \mathrm{Be}$ data are determined by matching the production rate pattern of ${ }^{10} \mathrm{Be}$ with the ${ }^{14} \mathrm{C}$ production ${ }^{18}$. The increasing rates are $7.2 \times 10^{3}$ (atoms per $\mathrm{cm}^{2}$ per year/year) from $\mathrm{AD} 770$ to 785 , and $6.2 \times 10^{3}$ (atoms per $\mathrm{cm}^{2}$ per year/year] from AD 985 to 995 . The scale of the increase around AD 994 is 0.86 times as large as that around AD 775. This value is consistent with the ratio for ${ }^{14} \mathrm{C}$ events (0.6 times larger) because ${ }^{10} \mathrm{Be}$ data have a lower time resolution $(\sim 10$ years resolution) than that of ${ }^{14} \mathrm{C}$ data. If the causes of two events are different, the difference between the ratios of ${ }^{14} \mathrm{C}$ and ${ }^{10} \mathrm{Be}$ production rates is expected. This difference is occurred by energy spectrums or particle species of the origin events. From the consistency of increasing ratio of AD 775 and 994 between ${ }^{14} \mathrm{C}$ and ${ }^{10} \mathrm{Be}$, the cause of the two events must be same.

Possible causes of these ${ }^{14} \mathrm{C}$ events are large SPEs or cosmic gamma-ray events ${ }^{1}$. For gamma-ray events, there are supernova explosions and $\mathrm{GRBs}^{5}$. The supernova remnants corresponding to $\mathrm{AD} 775$ and 994 have not been detected (http:// hea-www.harvard.edu/ChandraSNR/snrcat_gal.html (Chandra Supernova Remnant Catalog) $)^{19}$ and historical documentation has not been found ${ }^{20}$. Therefore, a supernova origin is quite unlikely ${ }^{1,5}$. Although only the normal supernova origin was considered in Miyake et al. ${ }^{1}$, Hambaryan and Neuhauser claim that a short GRB $(<2 \mathrm{~s})$ can explain the AD 775 event $^{5}$. In case of a short GRB, its spectral hardness is consistent with the differential production rates of ${ }^{14} \mathrm{C}$ and ${ }^{10} \mathrm{Be}$, and the absence of historical records of a supernova or a supernova remnant is consistent with a short $\mathrm{GRB}^{5}$. Although they claim that the observed rate of short GRBs (one event in $3.75 \times 10^{6}$ years ${ }^{5}$ ) and that of ${ }^{14} \mathrm{C}$ events (one ${ }^{14} \mathrm{C}$ event in 3,000 years) are consistent within $2.6 \sigma$ (ref. 4), the finding of the second ${ }^{14} \mathrm{C}$ event makes a ${ }^{14} \mathrm{C}$ event rate large, and the consistency between the observed rate of short GRBs and the ${ }^{14} \mathrm{C}$ event rate becomes worse (the probability of a short GRB rate with one ${ }^{14} \mathrm{C}$ event in 1,500 years is $0.04 \%)$. Adding to this, it is possible that the ${ }^{14} \mathrm{C}$ event rate is larger because there are many periods without a 1 -year resolution measurement of ${ }^{14} \mathrm{C}$ content in the 3,000-year period. The actual ${ }^{14} \mathrm{C}$ event rate should be $1 / 800$ years (detected event/measured 
period with 1-2-year resolution), and the probability is $0.02 \%$. Melott and Thomas ${ }^{2}$ also discuss the event rate of short GRB (a probablility of order $10^{-4}$ over 1,250 years); however, their rate is lower than Hambaryan and Neuhauser. Then, the short GRB is less likely to be the cause. In addition, Hambaryan and Neuhauser claim that it is possible that more short GRBs exist than those observed, which would explain the inconsistency in the event rate between the observed short GRB rate and the ${ }^{14} \mathrm{C}$ event rate; however, additional studies are necessary to confirm this claim.

Next, we consider the SPE origin. An emergence of SPE is considered to be closely bound to solar activity, such as solar flares and CMEs. To know the solar activity during 8-10th centuries, we have examined the IntCal data set ${ }^{6}$. The variation of ${ }^{14} \mathrm{C}$ content (which is closely related to the flux of galactic cosmic rays reaching the earth) is mainly modulated by the solar magnetic activity and the geomagnetic field. The ${ }^{14} \mathrm{C}$ variation in the past several hundred years reflects the solar magnetic activity as indicated by the sunspot records. In particular, periods of solar inactivity known as grand solar minima can be identified as large peaks in the past ${ }^{14} \mathrm{C}$ content record. The period from the late 13th century to the early 19th century is known as 'little ice age', which includes the Wolf, Spörer, Maunder and Dalton solar minima. On the other hand, the $8-10$ th centuries have no grand solar minimum. There have been some attempts to reconstruct the solar activity by using ${ }^{14} \mathrm{C}$ data set ${ }^{21,22}$, and they showed higher solar activity levels during the 8-10th centuries than that during the 13-19th centuries, on average. This fact may explain why the $8-10$ th centuries have two rapid increases of ${ }^{14} \mathrm{C}$ content and after the 11th century there were no such events.

Based on a reassessment of an energy spectrum of SPE, production calculations of ${ }^{14} \mathrm{C}$ and ${ }^{10} \mathrm{Be}$, and a deposition model, Usoskin and Kovaltsov ${ }^{3}$ claimed that the AD 775 event can be explained by an extreme SPE that was about 50 times larger than the largest SPE in AD 1956. From this aspect, the AD 994 event is about 30 times larger than SPE 1956. The energetic level does not have a serious effect on living matter on the earth ${ }^{2}$. Also, according to Usoskin and Kovaltsov ${ }^{3}$, the occurrence rate of the SPE775 event is $10^{-4}$ per year and that of the SPE994 event is $10^{-3}$ per years. It is possible that these events occur within 200 years assuming that SPEs are mutually independent. Although they claimed that there is no apparent relationship between the occurrence of SPE and the solar activity level, or it is proposed that large SPEs are occurred more likely during grand solar minima ${ }^{23-25}$, we doubt these claims because the two events occurred in the non-solar minimum period.

Considering the ${ }^{14} \mathrm{C}$ event rate and higher solar activity in the $8-10$ th centuries, a solar origin is a plausible cause of ${ }^{14} \mathrm{C}$ increase events. Detection of the second ${ }^{14} \mathrm{C}$ event indicates the possibility that a lot of smaller ${ }^{14} \mathrm{C}$ increases are hidden in the periods when the ${ }^{14} \mathrm{C}$ content has not been measured with a 1 -year resolution. In the future, it will be necessary to conduct investigations of ${ }^{14} \mathrm{C}$ records during additional unmeasured periods with a 1-year resolution.

\section{Methods}

Sample preparation. To measure ${ }^{14} \mathrm{C}$ content in tree rings, we have to extract graphite from the wood samples. First, we separated each annual ring using a cutter knife. Then, we obtained cellulose, which does not move between rings after the rings are formed, by applying a chemical wash for each wood slice. The chemical wash consists of ultrasonic cleaning, acid-alkali-acid treatments, sodium chlorite treatment and neutralization process. The treated material was combusted to $\mathrm{CO}_{2}$ and purified in vacuum lines. Finally, purified $\mathrm{CO}_{2}$ is graphitized by hydrogen reduction under the catalytic influence of iron powder.
AMS measurement. We measured the ${ }^{14} \mathrm{C}$ content in extracted graphite using an accelerator mass spectrometer (AMS) at the Center for Chronological Research $^{26}$. As AMS provides a relative measurement, six standard samples (NIST SRM4990C oxalic acid, the new NBS standard) were measured in the same batch. Two blank samples were also measured to determine the background (commercial oxalic acid was obtained from Wako Pure Chemical Industries). The concentration of ${ }^{14} \mathrm{C}$ expressed as $\Delta^{14} \mathrm{C}$, which is the ageand isotopic fractionation-corrected value, was calculated according to the method by Stuiver and Polach ${ }^{7}$. The typical precision of ${ }^{14} \mathrm{C}$ content measurements was $2.8 \%$.

\section{References}

1. Miyake, F., Nagaya, K., Masuda, K. \& Nakamura, T. A signature of cosmic-ray increase in AD 774-775 from tree rings in Japan. Nature 486, 240-242 (2012).

2. Melott, A. L. \& Thomas, B. C. Causes of an $\mathrm{AD} 774-775{ }^{14} \mathrm{C}$ increase. Nature 491, E1-E2 (2012).

3. Usoskin, I. G. \& Kovaltsov, G. A. Occurrence of extreme solar particle events: assessment from historical proxy data. Astrophys. J. 757, 92, doi:10.1088/0004637X/757/1/92 (2012).

4. Gibbons, G. W. \& Werner, M. C. More medieval clues to cosmic-ray event. Nature 487, 432 (2012).

5. Hambaryan, V. V. \& Neuhäuser, R. A galactic short gamma-ray burst as cause for the ${ }^{14} \mathrm{C}$ peak in $\mathrm{AD} 774 / 5$. Mon. Not. R. Astron. Soc. 430, 32-36 (2013).

6. Reimer, P. J. et al. IntCal09 and marin09 radiocarbon age calibration curves, 0 50,000 years cal BP. Radiocarbon 51, 1111-1150 (2009).

7. Stuiver, M. \& Polach, H. A. Discussion: reporting of ${ }^{14} \mathrm{C}$ data. Radiocarbon 19, 355-363 (1977).

8. Stuiver, M. et al. INTCAL98 radiocarbon age calibration, 24,000-0 cal BP. Radiocarbon 40, 1041-1083 (1998)

9. Nakamura, T. et al. High precision ${ }^{14} \mathrm{C}$ measurements and wiggle-match dating of tree rings at Nagoya University. Nucl. Instr. Meth. B 259, 408-413 (2007).

10. Menjo, H. et al. In Proceedings of the 29th International Cosmic Ray Conference Vol 2 (ed Acharya, B. S.) 357-360 (Tata Institute of Fundamental Research, Mumbai, 2005).

11. Damon, P. E., Kaimei, D., Kocharov, G. E., Mikheeva, I. B. \& Peristykh, A. N. Radiocarbon production by the gamma-ray component of supernova explosions. Radiocarbon 37, 599-604 (1995).

12. Stuiver, M., Reimer, P. J. \& Braziunas, T. F. High-precision radiocarbon age calibration for terrestrial and marine samples. Radiocarbon 40, 1127-1151 (1998).

13. McCracken, K., McDonald, F., Beer, J., Raisbeck, G. \& Yiou, F. A. Phenomenological study of the long-term cosmic ray modulation, 850-1958 AD. J. Geophys. Res. 109, A12103 (2004).

14. Delaygue, G. \& Bard, E. An Antarctic view of Beryllium-10 and solar activity for the past millennium. Clim. Dyn. 36, 2201 (2011).

15. Miyahara, H., Masuda, K., Muraki, Y., Kitagawa, H. \& Nakamura, T. Variation of solar cyclicity during the Spoerer Minimum. J. Geophys. Res. 111, A03103 (2006).

16. Miyahara, H. et al. Is the sun heading for another Maunder minimum? Precursors of the grand solar minima. J. Cosmol. 8, 1970-1982 (2010).

17. Miyahara, H. et al. Cyclicity of solar activity during the Maunder Minimum deduced from radiocarbon content. Sol. Phys. 224, 317-322 (2004).

18. Horiuchi, K. et al. Ice core record of ${ }^{10} \mathrm{Be}$ over the past millennium from Dome Fuji, Antarctica: a new proxy record of past solar activity and a powerful tool for stratigraphic dating. Quat. Geochronol. 3, 253-261 (2008).

19. Green, D. A. A revised Galactic supernova remnant catalogue. Bull. Astr. Soc. India 37, 45-61 (2009).

20. Stephenson, F. R. A revised catalogue of pre-telescopic galactic novae and supernovae. Q. Jl R. Astr. Soc. 17, 121-138 (1976).

21. Solanki, S. K., Usoskin, I. G., Kromer, B., Schuessler, M. \& Beer, J. An unusually active Sun during recent decades compared to the previous 11,000 years. Nature 431, 1084-1087 (2004).

22. Usoskin, I. G., Solanki, S. K. \& Korte, M. Solar activity reconstructed over the last 7000 years: The influence of geomagnetic field changes. Geophys. Res. Lett. 33, L08103 (2006).

23. Barnard, L. \& Lockwood, M. A survey of gradual solar energetic particle events. J. Geophys. Res. 116, A05103 (2011).

24. Barnard, L. et al. Predicting space climate change. Geophys. Res. Lett. 38, L16103 (2011).

25. Owens, M. J., Lockwood, M., Barnard, L. \& Davis, C. J. Solar cycle 24: Implications for energetic particles and long-term space climate change. Geophys. Res. Lett. 38, L19106 (2011).

26. Nakamura, T. et al. The HVEE Tandetron AMS system at Nagoya University. Nucl. Instrum. Methods B 172, 52-57 (2000). 


\section{Acknowledgements}

We thank K. Kimura for dating the sample tree rings by dendrochronology. We also thank $\mathrm{Y}$ Itow, H. Tajima, Y. Matsubara and T. Sako for commenting on our manuscript. This work was partly supported by Grants-in-Aid for Scientific Research (B:22340144) provided by the Ministry of Education, Culture, Sports, Science and Technology (MEXT) of Japan. F.M.'s work is supported by a Research Fellowship of the Japan Society for the Promotion of Science.

\section{Author contributions}

F.M. prepared samples. T.N. measured ${ }^{14} \mathrm{C}$ content by AMS at Nagoya University. F.M. and K.M. discussed the results. F.M. prepared the manuscript. K.M. commented on the manuscript.

\section{Additional information}

Supplementary Information accompanies this paper at http://www.nature.com/ naturecommunications

Competing financial interests: The authors declare no competing financial interests..

Reprints and permission information is available online at http://npg.nature.com/ reprintsandpermissions/

How to cite this article: Miyake, F. et al. Another rapid event in the carbon-14 content of tree rings. Nat. Commun. 4:1748 doi: 10.1038/ncomms2783 (2013). 


\section{Corrigendum: Another rapid event in the carbon-14 content of tree rings}

Fusa Miyake, Kimiaki Masuda \& Toshio Nakamura

Nature Communications 4:1748 doi:10.1038/ncomms2783 (2013); Published 23 Apr 2013; Updated 7 Nov 2013

The original version of this Article contained a chronological error concerning the counting of tree ring layers. During reanalysis of the data after publication, a missing layer was discovered around AD 956, which meant that all dates derived from annual layer counts beyond this datum needed to be increased by 1 year. For example, the date ranges quoted in the Abstract required revision from 'AD 822 to 1020' and 'AD 992 to 993' to 'AD 822 to 1021' and 'AD 993 to 994', respectively. These changes have now been applied throughout the PDF and HTML versions of the Article and accompanying Supplementary Information. 